\title{
Survival analysis—part 1
}

\author{
Salil Vasudeo Deo ${ }^{1,2} \cdot$ Vaishali Deo $^{1} \cdot$ Varun Sundaram $^{1}$ \\ Received: 4 August 2020 / Revised: 30 August 2020 / Accepted: 4 September 2020 / Published online: 2 October 2020 \\ (C) This is a U.S. Government work and not under copyright protection in the US; foreign copyright protection may apply 2020
}

\section{Introduction}

The area of statistics that deals with analyzing results associated with a "time" component is called survival analysis. For example, calculating the percentage of patients that are alive 10 years after coronary artery bypass surgery (CABG) or understanding the differences in 10-year survival between patients receiving mechanical or bio-prosthetic aortic valve are questions which can be answered by applying survival analysis techniques.

So, from our earlier examples, it is evident that survival analysis is a field of statistics that deals with analyzing data that has a temporal component associated with it. This brief review is structured as follows: (1) Unique nature of survival data; concept of censoring; (2) definitions important in understanding survival methods; (3) the Kaplan and Meier survivor method.

\section{Why do we need different techniques to analyze this type of data?}

The analysis of survival data needs special methods because:

1 The data contains a start time. The start time depends upon the question we need to answer in our study. Common examples are (a) determine survival following a surgical procedure, where the surgery date is the starting point. (b) Understand the natural history of a disease process where the date of diagnosis can be the start time. So, to obtain a

Salil Vasudeo Deo

svd14@case.edu

1 Cleveland VA Medical Center, North-east Ohio Veteran Affairs Health-care System, Cleveland, $\mathrm{OH}$ 44106, USA

2 Case School of Medicine, Case Western Reserve University, Cleveland, OH 44106, USA meaningful understanding of our data, we need to know the exact time point when each subject enters the study. This is the point at which the subject is now at risk of developing the endpoint of interest. However, the unique nature of survival data means that patients entering the study have different start time depending upon when they had the surgery or were diagnosed with the disease. The patients will then be followed from that point onwards.

2 We now present these aspects of survival data by considering a simple example. In our study (Fig. 1), we follow up subjects for a specific period of time. This time period can be calculated in any unit (days, months, or years). However, at some point, our follow-up ends. We have developed a prospective study to enroll patients undergoing CABG to investigate long-term mortality. The enrollment phase (01/01/2010-31/12/2014) will involve recruiting patients that consent to be part of our study. The $\mathrm{x}$-axis depicts the timeline of our study. As shown, in the enrollment phase (01/01/2010-31/12/2014), patients A-D have entered the study. They enter the study on the day they are operated. From that time point, they are considered to be "at risk" for incurring the outcome of interest. (This concept of being "at risk" for the event is central to survival analysis and will be discussed further.) After the enrollment period is over, the patients are still followed up and the study ends on 31/12/2018. This leads us to the second important term regarding survival analysis, i.e., censoring. Censoring is defined as the way to account for those patients that are alive or lost to followup and hence have an incomplete follow-up time [1]. In our study, patients B and C died. Hence, there is no confusion regarding their duration of survival. It corresponds to the drawn line segment in front of their names. However, for patients A and D, we need to think. Patient A was alive when we last met at a follow-up visit few months ago; however, we do not have any information regarding him since that visit. Patient $\mathrm{D}$, we know, is alive today. While we know that patient $\mathrm{D}$ would eventually die, the date of death is unclear as it would happen after the study end date $(31 / 12 / 2018)$. Thus, our information 
Fig. 1 This figure presents a hypothetical example of 4 patients included in a study to evaluate survival after coronary artery bypass grafting. As discussed in the paper, each patient may enter the study at different calendar time points, stay in the study for differing durations, and leave the study either because the patient dies or is alive at the end of follow-up.

Briefly, these are the unique challenges inherent in the analysis of time-related information

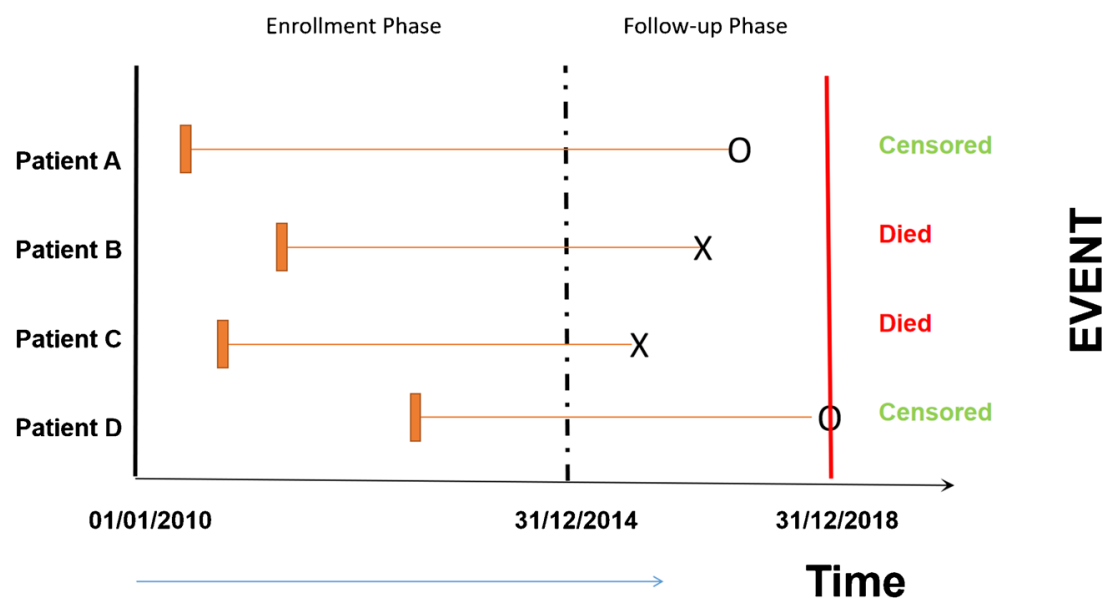

regarding $\mathrm{A}$ and $\mathrm{D}$ is incomplete and by definition they are censored at their last date of follow-up. More accurately, A and $\mathrm{D}$ are right censored at their last date of follow-up. Right censoring is defined as the phenomenon where a subject leaves the study before the endpoint of interest occurs, or the study ends before the endpoint has occurred.

\section{The survivor and hazard function}

Survival, hazard, and cumulative hazard: When we have a few patients, long-term survival can be reported with descriptive methods. However, with a few hundred or more patients and varying follow-up times, we need some more definitions and corresponding mathematical equations to understand our data and interpret our results. Below, we present some key terms with equations, but attempt to explain them as plainly as our understanding allows us to. However, we need to initially define some key elements:

$T$ random variable for a patient's survival time

$t$ actual time value of interest

Survivor function $S(t)$ : The survivor function is:

$S(t)=\operatorname{Pr}(T>t)$ where $0 \leq t \leq$ Infinity.

To interpret, the survivor function is the probability of being alive for a particular time " $t$ " that we are interested in understanding and reporting. In our study, that could be $1,3,5$, or 10 years or even longer. $S(t)$ is a probability; hence, it is always a positive value and is always $0 \leq S(t) \leq$ 1. As the time " $t$ " increases, $S(t)$ can remain constant or decrease; however, it can never increase. The survivor function is the most common way of reporting survival data. In a later section, we will discuss how it is calculated and how it can be meaningfully interpreted.

For example, if we want to estimate the probability of being alive at the end of 5 years after CABG, we would be estimating the survivor function.

Hazard function $h(t)$ : The hazard function is:

$$
h(t)=\lim _{\Delta t \rightarrow 0} \frac{S(t)-S(t+\Delta t)}{\Delta t}
$$

Here:

$T$ actual survival time

$t$ the time " $t$ " for which the hazard rate is calculated

$\Delta t$ a small increase in time " $t$ "

The hazard function is simply the instantaneous risk, force of mortality, or instantaneous failure rate at a specific time point " $t$ " during the whole time of follow-up [2]. We need to understand a few more key aspects of the $h(t)$ before we proceed to understand the relationship between $S(t)$ and $h(t)$. Looking closely at the formula for $h(t)$, we notice that it is a fraction, i.e., it contains a numerator and denominator. The numerator is in fact $S(t+$ delta $t)-S(t)$, i.e., what is the difference in survival between two closely separated time points and the denominator is the time difference "delta $t$." Hence, some key aspects of $h(t)$ are it is a rate, unlike $S(t)$, it is not bound between 0 and 1 , and the value depends upon the unit used to measure time.

For example, if want to understand if the risk of death changes after $\mathrm{CABG}$ during first year, we would estimate the hazard rate for our group of patients. The hazard rate will depend upon the unit of time (day, week, month) used in the analysis.

We can now graph the corresponding survivor and hazard function for our dataset. Figure $2 \mathrm{~A}$ is a plot that graphs the survivor function in our data. The $\mathrm{x}$-axis is the time of followup; in this case, it is measured in days. The y-axis is the probability of survival at that time corresponding to the $\mathrm{x}$ - 
Fig. 2 These graphs depict the survival function $[S(t)]$ and the corresponding hazard rate $[h(t)]$ for the same data set. The line which depicts the survival changes according to the observed hazard rate at that time

\section{A Plot of the Survivor function $\mathbf{S}(t)$} for our data

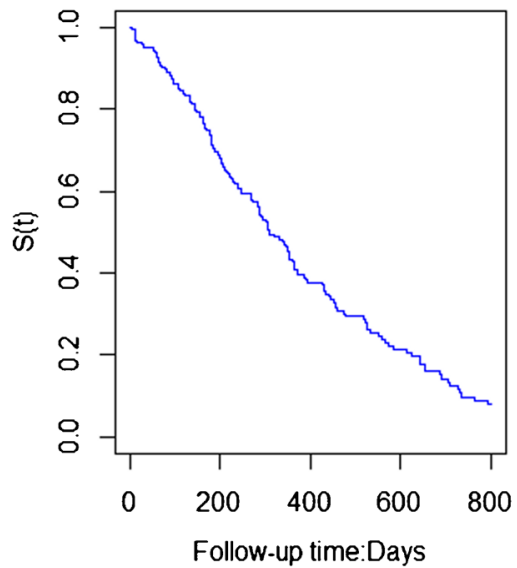

B Plot of Hazard function $h(t)$ for our data

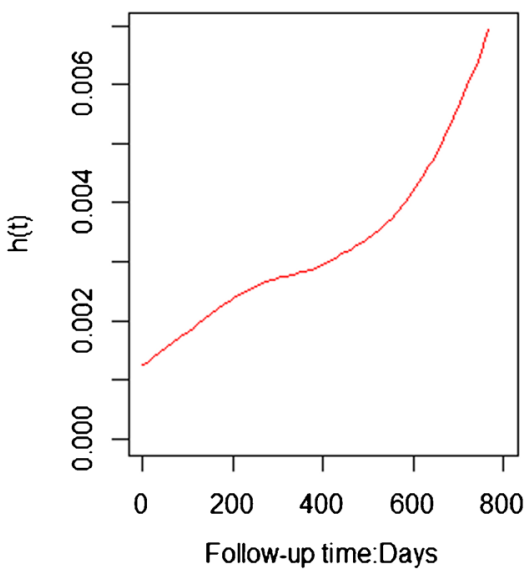

axis. This graph fulfills all the elements of $S(t)$ that we discussed earlier, and it starts at 1 , it subtends towards 0 , it does not increase at any time, and lastly in this case, it does not reach 0 at the end of follow-up. This is due to the censoring that we defined earlier. Figure 2B provides the corresponding hazard function $h(t)$ for our data. As seen, the hazard is continuously increasing throughout the follow-up period. The hazard for mortality appears to increase very rapidly from time $600-800$ days during follow-up. However, as seen in Fig. 3, survival between data sets can differ depending upon the time of follow-up, and the number of patients that die and are censored during that period. As these two data sets have different $S(t)$, they correspondingly have very different $h(t)$. Clearly, patients in the first data set have higher mortality with very few remaining alive at 800 days. Compare that with the second data, where approximately 0.6 remain alive or are censored at 800 days. This difference is mirrored in the very separate trajectories of the hazard plot for these two data sets (Fig. 4).

While both $S(t)$ and $h(t)$ are important in understanding our data, most published studies in cardiovascular journals will focus on the survival plots to explain their group survival. Along with being easy to understand and visually compare, the hazard plot requires post-processing

Plot of $S(t)$

for two Data sets

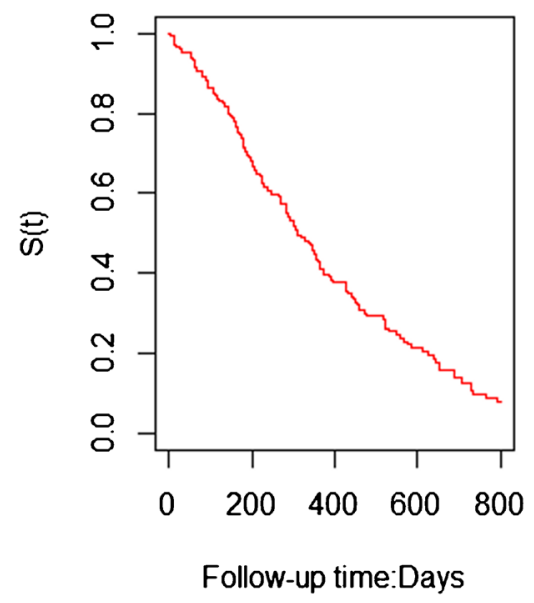

Fig. 3 These two graphs depict the survival function for very different group of patients. The patients in the first graph appear to die at very regular intervals. The initial line shows a pretty rapid decline demonstrating a high hazard rate, which then appears to reduce after approximately 400 days of follow-up. At the end of follow-up, approximately $10 \%$ patients are estimated to be living. The second graph depicts a very different picture. Here, survival appears to be good

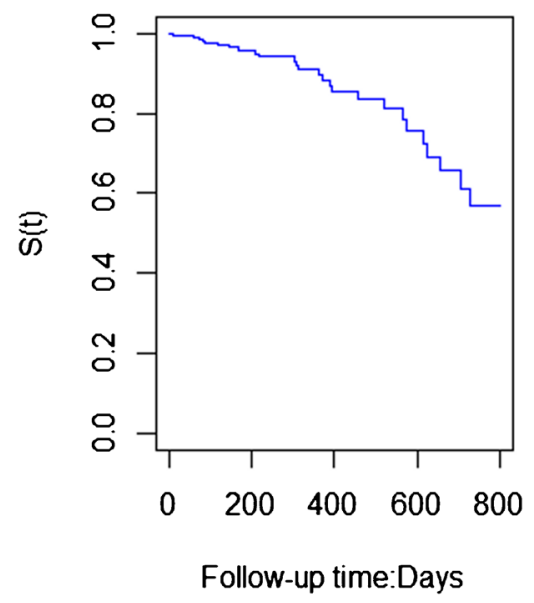

with a low hazard rate. The survival curve then declines faster after around 400 days, which may be due to an increasing hazard rate. At the end of follow-up, overall survival is still pretty good with approximately $60 \%$ surviving. Another important point to understand here is that the survival function $[S(t)]$ is always limited between $(1,0)$. As explained in the paper, it starts at 1 and then either remains constant or declines during the follow-up period 
Fig. 4 These graphs depict the corresponding hazard function $[h(t)]$ for the same group of patients whose survival function $[S(t)]$ is graphed in Fig. 3. As Fig. 3 shows, it is very clear how different these two groups of patients are with regard to their hazard function $[h(t)]$. Like the survival function $[S(t)]$, the hazard rate $h(t)$ is also always positive; however, unlike $S(t)$, it can increase or decrease during the follow-up time
Plot of $h(t)$

for two Data sets
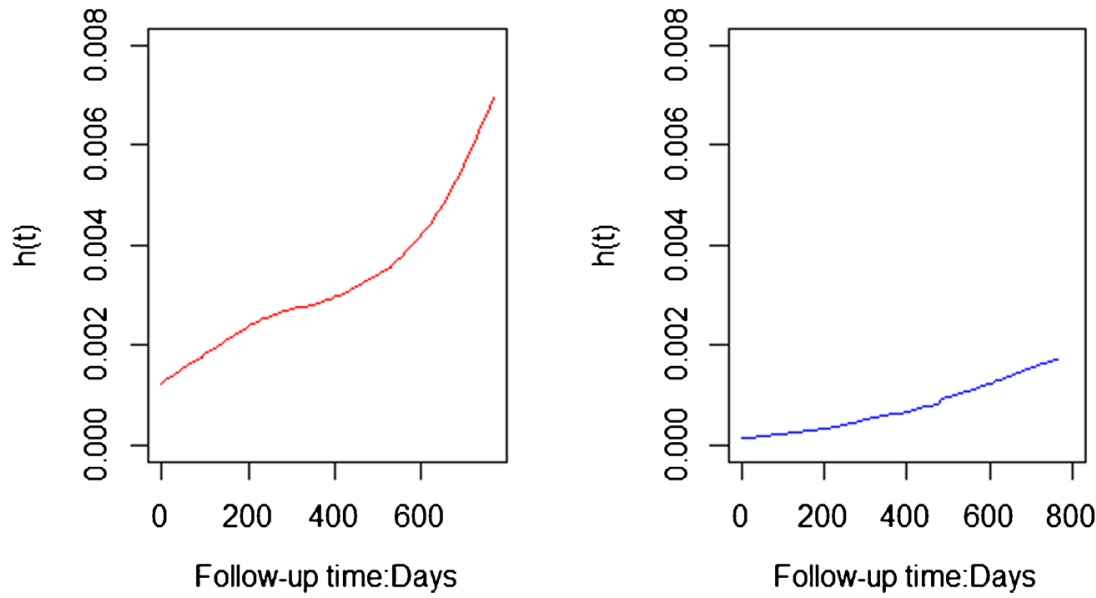

of estimates to achieve the smooth curves that are depicted in Figs. 3 and 4. This smoothing parameter can change the appearance of the hazard plots. Thus, survivor plots remain the most common method to graphically demonstrate time-related events in medical literature. Apart from obtaining survival estimates for the whole cohort, we can easily obtain survival probabilities to compare patients within certain groups for example male vs female, patients with diabetes mellitus and without diabetes mellitus, or according to procedure like off-pump CABG vs conventional CABG. Estimation of the survivor function and cumulative failure is achieved using many methods. Among them, the most often used to calculate the survival function and referenced in surgical literature is the Kaplan and Meier method.

The Kaplan and Meier method:

Kaplan and Meier (1958) introduced their method of calculating the survival function for censored data (Table 1).

The above table and Fig. 5 provide an understanding of how the Kaplan Meier (KM) survival is calculated. The steps are as follows: (a) Arrange patients from the smallest to largest time periods.

(b) At each time period that a death occurs, obtain the number of patient alive at the start and end of that time period.

(c) The Kaplan Meier estimator is now obtained as the product of the failure in that period and the survival of the earlier period. Hence, it is also called a product-limit survival estimator.

(d) Hence, each failure contributes to a step down with the actual drop depending upon the number of patients that were lost during that period. Patients censored also contribute to the calculation as:

Number of patients at the end of that period

$=($ Number of patients at the start)

$$
-[(\text { Died })+(\text { Censored })] \text {. }
$$

Number of patients at the end of that period $=$ (Number of patients at the start $)-[($ Died $)+($ Censored $)]$.

Table 1 This table provides the calculations of hypothetical data graphed in Fig. 5. The Kaplan Meier survival estimator is calculated at each time point when death or occurs. The actual duration between times points $0,1,2,3,4$, and 5 may vary

\begin{tabular}{lllllll}
\hline Time & Start & Died & Censored & End & Survival & Survival calculation explained \\
\hline 0 & 10 & 0 & 0 & 10 & 1.000 & $=10 / 10$ \\
1 & 10 & 2 & 0 & 8 & 0.900 & $=(10 / 10) \times(8 / 10)$ \\
2 & 8 & 1 & 1 & 6 & 0.675 & $=(10 / 10) \times(8 / 10) \times(6 / 8)$ \\
3 & 6 & 2 & 0 & 4 & 0.450 & $=(10 / 10) \times(8 / 10) \times(6 / 8) \times(4 / 6)$ \\
4 & 4 & 1 & 1 & 2 & 0.225 & $=(10 / 10) \times(8 / 10) \times(6 / 8) \times(4 / 6) \times(2 / 4)$ \\
5 & 2 & 2 & 0 & 0 & 0.000 & $=(10 / 10) \times(8 / 10) \times(6 / 8) \times(4 / 6) \times(2 / 4) \times(0 / 2)$ \\
\hline
\end{tabular}




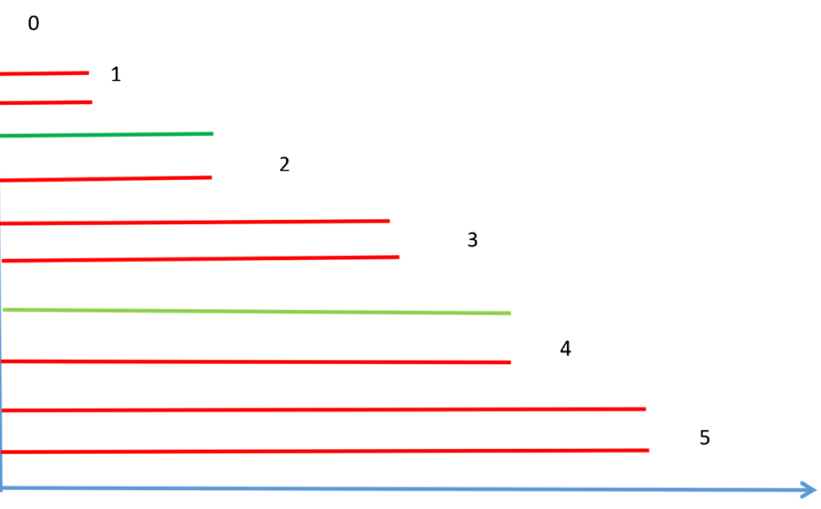

Time

Fig. 5 This figure with the accompanying Table 1 provides a better understanding regarding the Kaplan and Meier survival estimator. Patients are arranged according to their time spent in the study from smallest to largest time intervals. The time segments for patients that observed the event during follow-up are colored red, while those for censored patients are depicted in green. Calculations for the Kaplan and Meier estimate are performed at each time point that an event occurs. However, as demonstrated in the table, the survival estimate at the end of each time point is a product of the failure during that time interval and the survival estimate at the start of that time interval

The Kaplan Meier function is a very important tool to report the survival and cumulative failure for an event. It can also be reported and plotted for groups which can then be statistically compared. Thus going back to our introduction, the Kaplan Meier method would likely be among our first statistical analyses to report and observe long-term survival after $\mathrm{CABG}$, or to compare survival after mechanical vs bioprosthetic valve replacement.

\section{Conclusion}

This first part provides you with an understanding of how time to event data is different from other data. We also hope that we provided a clearer understanding of terms often used in survival analysis and the Kaplan Meier method.

In the next part, we will study further details regarding the presentation of Kaplan Meier survival and cumulative failure plots. We will also review statistical methods used to compare estimated survival between groups.

\section{Compliance with ethical standards}

Conflict of interest The authors declare that they have no conflict of interest.

\section{References}

1. Hosmer DW, Lemeshow S, May S. Applied survival analysis : regression modeling of time -to -event data. 2nd ed: Wiley Lifesciences; 2008.

2. Kleinbaum DG, Klein M. Survival analysis. a self-learning text. 3rd ed: Springer Verlag; 2012.

\section{Learning objectives}

1. To understand how time to event outcome data differs from other conventional data obtained from observational or randomized trials, hence the need for special statistical methods to deal with such data.

2. To learn the terms that are used to describe time-related outcome data.

3. To introduce the Kaplan and Meier estimator.

Publisher's note Springer Nature remains neutral with regard to jurisdictional claims in published maps and institutional affiliations. 